\title{
Development of Buffalo Hump in the course of antiretroviral therapy including raltegravir and unboosted atazanavir: a case report and review of the literature
}

\author{
Giancarlo Ceccarelli ${ }^{*}$, Gabriella d'Ettorre ${ }^{1}$, Francesco Marchetti ${ }^{2}$, Cecilia Rizza $^{1}$, Claudio M Mastroianni ${ }^{1}$,
} Bruno Carlesimo ${ }^{2}$, Vincenzo Vullo ${ }^{1}$

\begin{abstract}
Introduction: The availability of raltegravir plus atazanavir provides an alternative antiretroviral strategy that may be equally efficacious and less toxic than those currently recommended in HIV treatment guidelines. In fact, this new combination antiretroviral therapy attracts the attention of the scientific community because both drugs have a good safety profile coupled with potent antiviral activity, and their combined use would avert nucleoside- and ritonavir-related toxicities.

Case presentation: We describe the case of a 47-year-old, Caucasian woman treated for HIV-1 infection who developed Buffalo Hump during antiretroviral therapy, including raltegravir and unboosted atazanavir. Clinical evaluation and an ultrasonography scan of the cervical region showed a new progressive increase of lipohypertrophy and the results of DEXA confirmed these data. In our patient the worsening of the Buffalo Hump cannot be attributed to hypercortisolism; insulin-resistance, diabetes, dyslipidemia, hyperlactatemia and metabolic syndrome were not present. Moreover, she was not in therapy with antiretroviral drugs that are described as the cause of Buffalo Hump; on the other hand she developed this side effect three months after the switch of the antiretroviral therapy to raltegravir plus unboosted atazanavir.

Conclusion: Current data indicate that the etiology of HIV-associated Buffalo Hump remains elusive but is likely multifactorial; a possible contributing cause, but not the main cause, could be exposure to antiretroviral drugs. To the best of our knowledge, this is the first report on development of Buffalo Hump in the course of antiretroviral therapy, including the use of these drugs. On the basis of our data we can formulate the hypothesis of a pharmacological pathogenesis that underlies the development of this case of Buffalo Hump in the absence of other risk factors.
\end{abstract}

\section{Introduction}

Antiretroviral (ARV) treatment guidelines currently recommend ARV regimens containing a Nucleos(t)ide Reverse Transcriptase Inhibitors (N(t)RTIs) based backbone with a Non Nucleoside Reverse Transcriptase Inhibitor (NNRTI) or ritonavir boosted Protease Inhibitor (PI/r). However, significant toxicity has been associated with $\mathrm{N}(\mathrm{t}) \mathrm{RTI}(\mathrm{s})$ and $\mathrm{PI} / \mathrm{r}$ containing regimens. Recent

\footnotetext{
* Correspondence: giancarlo.ceccarelli@uniroma1.it

'Department of Infectious Diseases and Public Health, "Sapienza" University, Rome, Italy

Full list of author information is available at the end of the article
}

data presented by Gupta et al. show that the combination of raltegravir (RAL) plus unboosted atazanavir (ATV) may be an alternative effective ARV regimen demonstrating good virologic and immunologic response. Furthermore, the combination is well tolerated and has a low incidence of adverse effects [1]. Moreover, side effects reported by Zhu et al. during a study in healthy subjects were generally "mild-to moderate" in intensity. Common side effects seen when both drugs were taken were jaundice and headache [2]. Ripamonti et al. evidenced that after five to seven months of therapy based on RAL plus ATV no patients discontinued

\section{(Ciomed Central}


treatment due to drugs used in therapy, adverse events, and no one had a grade 3 or 4 lab toxicity [3]. For these reasons this combination of antiretroviral therapy based on RAL plus ATV attracts the attention of the scientific community because both drugs have a good safety profile coupled with potent antiviral activity, and their combined use would avert nucleoside- and ritonavir-related toxicities.

\section{Case presentation}

We describe the case of a 47-year-old, Caucasian woman treated for HIV-1 infection, who developed buffalo hump during antiretroviral therapy consisting of RAL and unboosted ATV. She was diagnosed with HIV disease in February 1999: the CD4+ cell count was 214/ mm3 (11\%) and the HIV viral load was 253,200 copies/ $\mathrm{ml}$ at that stage. An initial highly active antiretroviral therapy (HAART) regimen consisted of zidovudine, lamivudine and indinavir. After 18 months, the therapy was changed to stavudine, lamivudine and nevirapine because of an episode of acute renal colic. Our patient attended outpatient clinics on a regular basis, and was found to have a good immunological and virological response to HAART. By July 2004, she presented with a progressive peripheral fat loss; facial lipoatrophy was apparent but not severe. For these reasons the HAART combination was changed (November 2004), and stavudine was replaced by a nucleotide analogue tenofovir. The CD4+ count was 599/mm3 (16\%) and the HIV viral load was $<50$ copies $/ \mathrm{ml}$ at the change of the antiretroviral medications. She was on nevirapine and lamivudine plus tenofovir for five years with a good immune-virological response. We observed neither other fat accumulation nor fat loss and no significant metabolic disorders after the switch. Body Mass Index (BMI), glucose, cholesterol, triglyceride, plasma cortisol and insulin concentrations were normal.

In November 2009 the patient presented with a HIV viral load of 1551 copies $/ \mathrm{ml}$; a subsequent test of resistance showed the presence of K65R, K103S, M184V, and G190A. Because she refused therapies with an increased risk of metabolic alterations, the antiviral treatment was changed to RAL $400 \mathrm{mg}$ with unboosted ATV 200 mg twice daily.

Three months later, it was noted that she developed a new progressive increase of lipohypertrophy of the dorso-cervical region of the neck. There was no localized accumulation of fat in her abdomen and in the submental region of her neck. The hump in the back of her neck was causing neck pain, headaches off and on and sleep apnea. It was causing her discomfort and affecting the motion of her neck. An ultrasonography scan of the cervical region reported a large amount of subcutaneous fat around the posterior aspect of the neck (Figure 1). The results of DEXA confirmed these data. Fasting lipid profile showed a total Cholesterol of $170 \mathrm{mg} / \mathrm{dl}, \mathrm{HDL}-\mathrm{Cholesterol} 42 \mathrm{mg} / \mathrm{dl}$ and Triglycerides $148 \mathrm{mg} / \mathrm{dl}$. Fasting plasma glucose and response to a glucose tolerance test were normal. Her waist circumference was $80 \mathrm{~cm}$ and her BMI was 21. Moreover, there were no significant changes noted in diet, physical activity, and body weight. Thyroid hormones, plasma insulin, cortisol, estradiol, progesterone, prolactin, luteinizing and folliclestimulating hormone concentrations were normal. Her CD4-lymphocyte count was $844 / \mathrm{mm} 3$ (20\%) and HIV viral load was < 50 copies/ml.

In the next three months she developed a massive lipohypertrophy of the dorso-cervical region of her neck. For this reason the plastic surgery staff proposed surgical removal of the $\mathrm{BH}$ due to discomfort, localized pain and the progressive increase of lipohypertrophy of the dorso-cervical region of the neck. The patient refused this option and six months later we observed a stabilization of the subcutaneous fat of the cervical region.

\section{Discussion}

Buffalo Hump is commonly reported in adults with HIVassociated lipodystrophy. Accumulation of fat over the dorso-cervical spine is reported in $2 \%$ to $13 \%$ of HIV infected patients with a higher prevalence (6 to $13 \%$ ) in those showing any other feature of the lipodystrophy syndrome [4]. The pathogenesis underlying this aspect of lipodystrophic syndrome is poorly understood. Guallar et al. reported that Buffalo Hump adipose tissue shows specific disturbances in gene expression with respect to subcutaneous fat from HIV-1-infected/HAART-treated

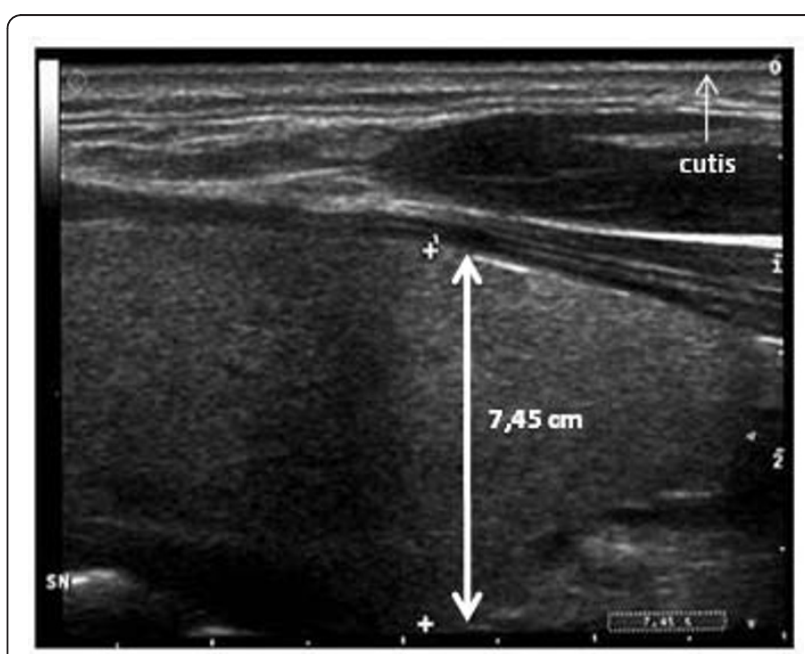

Figure 1 Ultrasonography scan of the cervical region. The scan evidenced a massive lipohypertrophy of the dorso-cervical region of the neck (the maximum diameter identified by focusing up and down through the planes of the section was $7.45 \mathrm{~cm}$ ). The area of fat accumulation was extended over the entire cervical region. 
patients [5]. Some reports indicate that Buffalo Hump is associated with other physical features of the lipodystrophy phenotype and suggest that hyperinsulinemia, insulin resistance, obesity, and hypercortisolism, are important components of this phenotype [6-9]. The close relationship between Buffalo Hump and glycaemic parameters suggests patients with Buffalo Hump are at higher risk for diabetes and metabolic syndrome. In fact, biochemically, patients with Buffalo Hump tend to have or develop signs or symptoms of metabolic syndrome. Mallon et al. reported that patients with Buffalo Hump had higher BMI and more total limb and abdominal fat than patients without Buffalo Hump. Current data indicate that a possible contributing cause, but not the main one, could be exposure to antiretroviral drugs: risk factors for Buffalo Hump are longer duration of use of protease inhibitors and longer duration of use of zidovudine [10]. Palacios et al. showed that Buffalo Hump was associated with treatment with saquinavir, indinavir, efavirenz, tenofovir and stavudine. Moreover, time of exposure to stavudine and fat loss, one of stavudine's major side-effects, were associated with Buffalo Hump [11]. Previous reports, however, indicated that the appearance of buffalo hump could not be associated with any specific component of HAART regimes and that it is associated with specific disturbances in gene expression of adipose tissue $[4,5]$.

We report the development of the Buffalo Hump cannot be attributed to hypercortisolism; insulin resistance, diabetes, dyslipidemia, hyperlactatemia and metabolic syndrome were not present. Moreover, there were no significant changes noted in our patient's diet, body weight and BMI. Her lifestyle was normal and she followed a regular exercise program.

At the moment she is not in therapy with antiretroviral drugs that are described as the cause of Buffalo Hump; on the other hand she developed this side effect three months after the switch of the antiretroviral therapy to RAL plus unboosted ATV. A caveat of this report is that she had a history of exposure to antiretroviral drugs (Zidovudine, Stavudine, Indinavir) associated with the development of Buffalo Hump. This condition may have predisposed the patient to develop the disorder and could constitute a background that contributes to the final appearance of buffalo hump after raltegravir plus atazanavir treatment. Current data indicate that the etiology of HIV-associated Buffalo Hump remains elusive but is likely multifactorial and includes, metabolic disorders, genetic factors, receipt of ART and HIV infection itself [12].

\section{Conclusions}

The availability of RAL and ATV provides an alternative ARV strategy that may be equally efficacious and less toxic than those currently recommended in HIV treatment guidelines. However, there are few data in the literature available to date regarding such a combination. There are no publications today that describe a relationship between RAL and unboosted ATV therapy and the occurrence of Buffalo Hump. This is the first report on the development of Buffalo Hump in the course of antiretroviral therapy including these drugs: on the basis of our data we can formulate the hypothesis of a pharmacological pathogenesis that underlies the development of this case of Buffalo Hump in absence of other risk factors. More investigation is required to determine if RAL plus unboosted ATV is a safe alternative to RTV boosted PI based ARV strategies and if there are significant side effects related to this ARV treatment.

\section{Consent}

Written informed consent was obtained from the patient for publication of this case report and accompanying images. A copy of the written consent is available for review by the Editor-in-Chief of this journal.

\section{Author details}

'Department of Infectious Diseases and Public Health, "Sapienza" University, Rome, Italy. "Department of Plastic Surgery, "Sapienza" University, Rome, Italy.

\section{Authors' contributions}

GC has made substantial contributions to conception and design, acquisition of data, analysis and interpretation of data. GD was involved in drafting the manuscript or revising it critically for important intellectual content. FM and CR made substantial contributions to the acquisition of data. CMM, BC and W gave final approval of the version to be published. All authors have read and approved the final manuscript.

\section{Competing interests}

The authors declare that they have no competing interests.

Received: 5 February 2010 Accepted: 17 February 2011

Published: 17 February 2011

\section{References}

1. Gupta S, Lataillade M, Farber S, Kozal MJ: Raltegravir with unboosted atazanavir $300 \mathrm{mg}$ twice daily in antiretroviral Treatment-experienced partecipants. J Int Assoc Physicians AIDS Care 2009, 8:87-92.

2. Zhu L, Mahnke L, Butterton J, Persson A, Stonier M, Comisar W, Paneblianco D, Breidinger S, Zhang J, Bertz R: Pharmacokinetics and safety of twice-daily atazanavir $(300 \mathrm{mg})$ and raltegravir $(400 \mathrm{mg})$ in healthy subjects. Program and abstracts of the 16th Conference on Retroviruses and Opportunistic Infections Montreal, Canada; 2009, Abstract 696.

3. Ripamonti D, Maggiolo F, d'Avolio A, Cattaneo D, Bonora S, Di Perri G: Steadystate pharmacokinetics of Atazanavir (200 mg BID) when combined with Raltegravir (400 mg BID) in HIV-1 infected adults. Program and abstracts of the 10th International Workshop on Clinical Pharmacology of HIV Therapy Amsterdam, Nederland; 2008, Abstract 0-14.

4. Mallon PW, Wand H, Law M, Miller J, Cooper DA, Carr A: HIV Lipodystrophy Case Definition Study; Australian Lipodystrophy Prevalence Survey Investigators. Buffalo hump seen in HIV-associated lipodystrophy is associated with hyperinsulinemia but not dyslipidemia. J Acquir Immune Defic Syndr 2005, 38:156-162.

5. Guallar JP, Gallego-Escuredo JM, Domingo JC, Alegre M, Fontdevila J, Martínez E, Hammond EL, Domingo P, Giralt M, Villarroya F: Differential gene expression indicates that 'buffalo hump' is a distinct adipose tissue disturbance in HIV-1-associated lipodystrophy. AIDS 2008, 22:575-584. 
6. Palella FJ Jr, Chmiel JS, Riddler SA, Calhoun B, Dobs A, Visscher B, Kingsley L: A novel pattern of lipoaccumulation in HIV-infected men. JAMA 2006, 296:766-768.

7. Rodriguez de la Concepcion ML, Domingo JC, Domingo P, Giralt M, Villarroya F: Uncoupling protein 1 gene expression implicates brown adipocytes in highly active antiretroviral therapy-associated lipomatosis. AIDS 2004, 18:959-960.

8. Wohl DA, McComsey G, Tebas P, Brown TT, Glesby MJ, Reeds D: Current concepts in the diagnosis and management of metabolic complications of HIV infection and its therapy. Clin Infect Dis 2006, 43:645-653.

9. Saint-Marc T, Touraine JL: Buffalo hump in HIV-1 infection. Lancet 1998, 352:319-320

10. Mallon PW, Wand H, Law M, Cooper DA, Carr A: Factors associated with buffalo Hump in a cohort of patients with HIV associated lipodystrophy. Antivir Ther 2003, 8(Suppl. 1), (abstract 715) In: Program and Abstracts of the 2nd IAS Conference on HIV Pathogenesis and Treatment (Paris, France),

11. Palacios R, Galindo MJ, Arranz JA, Lozano F, Estrada V, Rivero A, Morales D, Asensi V, del Arco A, Muñoz A, Santos J: Cervical lipomatosis in HIVinfected patients: a case-control study. HIV Med 2007, 8:17-21.

12. Wierzbicki AS, Purdon SD, Hardman TC, Kulasegaram R, Peters BS: HIV lipodystrophy and its metabolic consequences: implications for clinical practice. Curr Med Res Opin 2008, 24:609-624.

doi:10.1186/1752-1947-5-70

Cite this article as: Ceccarelli et al:: Development of Buffalo Hump in the course of antiretroviral therapy including raltegravir and unboosted atazanavir: a case report and review of the literature. Journal of Medical Case Reports 2011 5:70.

\section{Submit your next manuscript to BioMed Central and take full advantage of:}

- Convenient online submission

- Thorough peer review

- No space constraints or color figure charges

- Immediate publication on acceptance

- Inclusion in PubMed, CAS, Scopus and Google Scholar

- Research which is freely available for redistribution

Submit your manuscript at www.biomedcentral.com/submit 